\title{
PROYECTOS DE IMPLEMENTACIÓN DE SISTEMAS DE EJECUCIÓN DE MANUFACTURA MES MODELO DE PLANIFICACIÓN DE ALCANCE APLICADO EN LA INDUSTRIA COLOMBIANA
}

\author{
IMPLEMENTATION PROJECTS MANUFACTURING EXECUTION SYSTEMS \\ MES MODEL SCOPE PLANNING APPLIED IN THE COLOMBIAN INDUSTRY
}

\author{
Ing. Fabian Alonso Lara Vargas*, MSc. Luis Alberto Esteban Villamizar**. \\ Ing. Rosa Liliana Tarazona Cáceres*. \\ *Facultad de Ingeniería Electrónica, Grupo de investigación Informática y Tecnologías. \\ Emergentes ITEM, Universidad Pontificia Bolivariana Sede Montería, Córdoba. \\ E-mail: fabian.lara@upb.edu.co,rosa.tarazona@upb.edu.co \\ **Universidad de Pamplona, CICOM. \\ Ciudadela Universitaria, Pamplona, Tel.: (+577) 5685303, Fax: (+577) 568 5303, Ext. 144. \\ E-mail: lesteban@unipamplona.edu.co
}

Resumen: Este artículo describe un modelo de planificación del alcance propuesto para proyectos de implementación de sistemas de ejecución de manufactura MES. El modelo fue desarrollado a partir de los conceptos de gestión de proyectos y de metodologías diversas, estándares y mejores prácticas de la implementación de sistemas MES, en respuesta a la problemática que suscita una deficiente definición del alcance y de requerimientos en este tipo de proyectos. El modelo se aplicó en un proyecto en la industria colombiana.

Palabras clave: Sistema de ejecución de manufactura, manufactura integrada por computador, planificación de recursos de la empresa.

\begin{abstract}
This paper describes a model scope planning proposed for projects of implementation of manufacturing execution systems. The model was developed as from the concepts of projects management and of various methodologies, standards and better practices of the implementation of systems MES, in response to the problems that a deficient definition of scope and of requests raises in this type of projects. The model was applied in a project in the Colombian industry.
\end{abstract}

Keywords: Manufacturing execute system, computer integrated manufacturing, enterprise resource planning.

\section{INTRODUCCIÓN}

El presente artículo describe un modelo para la planificación del alcance en proyectos de implementación de sistemas MES (del inglés, Manufacturing Execute System), validado mediante la aplicación en una empresa de la industria colombiana y comparándolo con otros proyectos similares previamente implementados.
Los sistemas de ejecución de manufactura MES se conciben como un nivel del modelo CIM (del inglés, Computer Integrated Manufacturing) que describe las actividades del flujo de trabajo necesarias para la producción, el manteniendo de sus registros y la optimización de sus procesos (ISA, 2010). MES lleva los datos en tiempo real del nivel de producción, al nivel de ERP (del inglés, Enterprice Resource Planning) para poder 
ser analizados por el nivel directivo y tomar decisiones operativas en menos tiempo, pudiendo así sortear de una forma más rápida interrupciones, paradas o imprevistos típicos de la producción industrial (Kozletsov, 2012) (Banerjee, 2013). Muchos proyectos de implementación de sistemas MES fracasan porque no fueron correctamente estructurados en su organización de trabajo por la dirección del mismo, pues esta solo se siente responsable de la asignación de recursos y personal, resultando en la mayoría de los casos en problemas de organización más que en problemas técnicos de implementación (Kletti, 2007). El presente modelo busca disminuir la probabilidad de fracaso en la implementación de sistemas MES, relacionados con el área de gestión de alcance, al brindar un procedimiento general de cómo planificar dicho alcance. En la primera sección de este artículo se presenta el modelo teórico propuesto. La segunda sección incluye la descripción detallada de las etapas del modelo. La tercera sección presenta la aplicación del modelo propuesto en un proyecto de la industria de alimentos. En la cuarta sección se presentan algunas discusiones propias del modelo propuesto $y$ finalmente se dan algunas conclusiones referentes al modelo propuesto.

\section{MODELO DE PLANIFICACIÓN DE LA GESTIÓN DEL ALCANCE}

La búsqueda, recopilación, clasificación y análisis de información provenientes de distintas fuentes de información primaria y secundaria relacionada con el tema de gestión de proyectos en el área de los sistemas MES, produjo la creación de un modelo de planificación constituido por:
a. Procesos
b. Principios
c. Documentos
d. Normas y estándares

Para la construcción del primer componente denominado procesos, se usaron las metodologías para la gestión de proyectos PMBOK (del inglés, Project Management Body of Knowledge PMBOK) (Project Management Institute, 2013), y PRINCE2 (del inglés, PRojects IN Controlled Environments) (Turley, 2009), la metodología para la implementación de sistemas MES White Paper 19 "Aplicando un enfoque por fases para un proyecto exitoso (MES)" (D E Anisimov, 2011) (Christine Lesher, 2006), el modelo de implementación de Riley Elliott (Elliott, 2013), la metodología de implementación de CimNet System (Ferrazzi, 2012), el modelo de implementación de Tata Consulting Services (Ferrazzi, 2012), el modelo de implementación de Knight y Lamb (Jason Knight, 2006), la metodología de implementación de França Giunchetti (Giunchetti, 2004) y el modelo de implementación global MES basado en metodología ágil (Shrivastava, 2013). Ver Fig. 1.

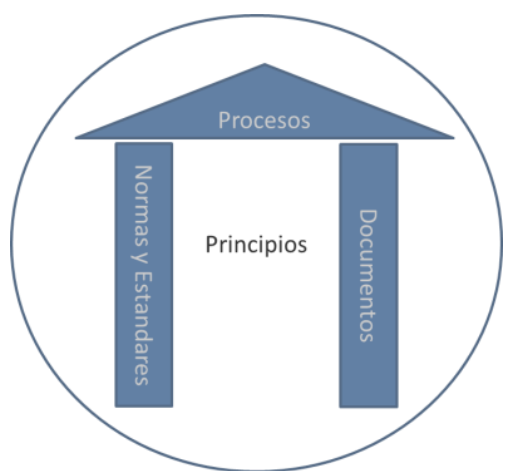

Fig. 1. Constitución del modelo de planificación. Fuente [Autor].

Los procesos que integran el modelo son los siguientes:

Proceso de justificación comercial: este proceso busca no solo dar inicio al proyecto, sino que además se tenga claridad por parte de los involucrados, de los beneficios del proyecto y que se pueda realizar un seguimiento de estos beneficios durante la ejecución de estos.

Proceso de lecciones aprendidas: este proceso se enfoca en revisar las lecciones aprendidas en otros proyectos similares para ponerlas en práctica en el proyecto a desarrollar.

Cabe resaltar que a pesar de que los procesos de justificación comercial y de lecciones aprendidas no se llevan a cabo en la planificación del alcance, sus productos si se deben tener en cuenta al momento de la elaboración de esta (Project Management Institute, 2013).

Proceso de gestión por excepción: en este proceso se describen cuales son parámetros de decisión del proyecto.

Proceso de gestión de productos del alcance: en este proceso se describe la forma de cómo se van a validar y controlar los productos del alcance.

Proceso de gestión de adaptabilidad y del cambio: en este proceso tiene como función ajustar los procesos administrativos al tamaño propio del proyecto, además de describir como 
se llevará a cabo la gestión de cambios en el proyecto.

Proceso de gestión de fases: este proceso permite la división del trabajo necesario para llevar a cabo los productos del alcance, a la vez que facilita la gestión de los requerimientos de los usuarios.

Por otro lado los principios que constituyen el modelo, fueron concebidos como elementos que deben tenerse en cuenta a la hora de planificar el alcance en cualquier proyecto de implementación de un sistema MES. La presentación de los mismos no obedece a un orden de implementación, ya que se encuentran elementos propios de la implementación y otros de la estructura del modelo.

- La elaboración de un documento sobre las razones de la ocurrencia de cambio para determinar la necesidad del cambio, el tipo de cambio y la prioridad del mismo (Project manager Alliance, 2013).

- Una deficiente especificación de requerimientos resulta en graves problemas al momento de realizar implementación de sistemas MES (Larsen, 2009).

- La clave de una implementación exitosa de MES, es la planificación completa desde que inicia hasta que termina el proyecto (Engelbrecht, 2008).

- Es fundamental definir que es MES para los interesados. El sistema MES debe estar siempre centrado en el negocio, no en el software MES (Clemons, 2013).

- Para la implementación de un sistema MES debe tenerse en cuenta la normatividad vigente para el sector industrial específico (Chris Wubbolt, 2012).

- La planificación del alcance en modelos de proceso incremental y evolutivo, se fundamenta en la retroalimentación de cada incremento para la definición del alcance del próximo incremento (Ailin Orjuela, 2011).

Para el componente denominado documentos, el modelo de planificación para la gestión de alcance establece los siguientes:

- Business case.

- Acta de inicio.

- Acta de lecciones aprendidas.

- Factores de adaptabilidad.

- Plan de excepciones.

- Definición del alcance.
- Plan de validación y control del alcance.

- Estructuras de desglose de trabajo.

- Plan de gestión de requisitos.

En el componente de normas y estándares, el modelo está compuesto por las siguientes; algunos de ellos son propios del área de gestión de proyectos, mientras que otros son usados en los sistemas de ejecución de manufactura:

- La normas ISA 95 (ISA, 2010).

- El Estándar de Práctica para Estructuras de Desglose de Trabajo (Practice Standard for work breakdown structures) del PMI (Project Management Institute, 2006).

- Las prácticas recomendadas para especificaciones de requerimientos de software IEEE (del inglés, Recommended Practice for Software Requirements Specifications) (The Institute of Electrical and Electronics Engineers, 2009).

\section{ETAPAS PARA LA IMPLEMENTACIÓN DEL MODELO DE PLANIFICACIÓN}

El modelo de planificación para la gestión del alcance, debe observarse como un macroproceso iterativo e incremental compuesto por las siguientes etapas, ver fig. 2 .

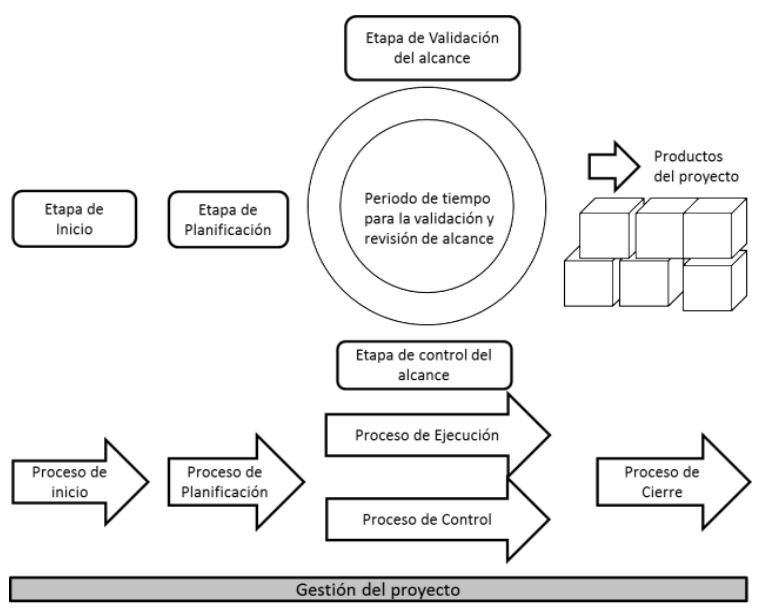

Fig 2. Descripción del modelo de planificación. Fuente: [Autor].

- Etapa de inicio: esta etapa se compone de los procesos de justificación comercial y lecciones aprendidas.

- Etapa de planificación del alcance: se compone de los procesos de gestión por excepción, gestión de productos del alcance, 
gestión de fases y gestión de adaptabilidad y del cambio.

- Etapa de validación del alcance: compuesta por el plan de validación del alcance.

- Etapa de control del alcance: está compuesta por el plan de control del alcance.

Una vez realizados todos los procesos de planificación, se prosigue con la ejecución del proyecto de implementación del sistema MES; en esta etapa se valida el alcance y entregan los productos requeridos por el proyecto a medida que el proyecto se desarrolle, la etapa de control del alcance, permite analizar desviaciones en el alcance del proyecto, para realizar las solicitudes de cambio y actualizar los documentos del proyecto si se requiere de ajustes. Por ello se deberán revisar nuevamente los documentos resultantes de los procesos de planificación para proceder con los cambios a que hubiere lugar para alcanzar los objetivos del proyecto y finalizar con el proceso de cierre del mismo.

\section{APLICACIÓN DEL MODELO EN LA INDUSTRIA COLOMBIANA}

El proceso de validación del modelo de planificación de la gestión de alcance, se hace mediante una prueba piloto de implementación en una máquina dispuesta para su instalación en una empresa del sector de alimentos. La prueba piloto presentó las siguientes características.

- Nombre del proyecto: Implementación de un secador de alimentos con sistema de control, SCADA y MES, aplicado al procesamiento de maíz.

- Empresa contratista: Ingeniería y Servicios Industriales SAS.

- NIT: 900504440-6.

- Empresa Contratante: No Menciona

- Monto: \$28.000.000

- Alcance: Diseño y montaje de un secador de alimentos con sistema de control, SCADA y MES, aplicado al procesamiento de maíz.

- Tiempo: 05/02/2015 - 17/04/2015

- Lecciones aprendidas: Véase discusiones.

- Metodología de gestión empleada: Modelo de planificación de la gestión de alcance.

Para la aplicación del modelo se establecieron reuniones cortas a la semana con el equipo de trabajo del proyecto para dar a conocer los avances, los retrasos, las oportunidades y las es trategias de mitigación. Se realizaron entrevistas y encuestas a los involucrados al finalizar el proyecto.

Según los resultados obtenidos se logró entregar un producto conforme, dentro de los tiempos y expectativas del cliente, aunque cabe resaltar que los tiempos del cliente, no coincidieron con el tiempo planificado, debido a demoras en la recepción del producto. El modelo permitió que se definiera de forma clara el alcance del producto y las restricciones del mismo, ya que en experiencias de proyectos anteriores, en áreas a fines al proyecto piloto, la empresa contratante y la contratista presentaron no conformidades con la definición del alcance.

\section{DISCUSIONES}

Teniendo en cuenta que según Bradenburg, un proyecto de implementación de sistemas MES se compone a su vez de tres subproyectos, los cuales estan en los niveles de instrumentación, control supervisión y sistemas MES (Brandenburg, 2013), se tomaron experiencias de los siguientes proyectos, para validar el modelo mediante la comparación de resultados a partir de entrevistas realizadas a los involucrados. Los proyectos tienen las siguientes características.

- Nombre del proyecto: Suministro e instalación de componentes para la construcción de un secador de alimentos.

- Empresa contratista: Ingeniería y Servicios Industriales SAS.

- NIT:900504440-6.

- Empresa Contratante: No Menciona.

- Monto: \$ 16.603 .779

- Alcance: Suministro e instalación de componentes de un secador de alimentos.

- Tiempo: 25/07/2012 - 17/12/2012

- Metodología de gestión empleada: Ninguna.

- Nombre del proyecto: Implementación de un Sistema MES, aplicación SCADA de la planta térmica de la Universidad Pontificia Bolivariana Seccional Bucaramanga.

- Empresa contratista: Ing Jesica Ariza Duran (UPB Bucaramanga).

- Empresa Contratante: No Menciona.

- Monto: No Menciona.

- Alcance: Diseño e implementación de un sistema MES aplicación SCADA de la planta térmica.

- Tiempo: 2012-2013 ( un año y medio )

- Metodología de gestión empleada: Ninguna. 
- Nombre del proyecto: Diseño e implementación de un sistema MES para la visualización de la velocidad promedio de producción utilizando el horno del Laboratorio de Control Digital de la Universidad Pontificia Bolivariana a través del software Labview ${ }^{\circledR}$ 2009.

- Empresa contratista: Rafael Arroyo.

- Empresa Contratante: No Menciona.

- Monto: No Menciona.

- Alcance: Diseño e implementación de un sistema MES en el horno del Laboratorio de Control Digital de la Universidad Pontificia Bolivariana a través del software Labview ${ }^{\circledR}$ 2009.

- Tiempo: 2012 - (actualmente).

- Metodología de gestión empleada: Ninguna.

Las lecciones aprendidas fueron implementadas en el modelo de planificación de la gestión de alcance.

\section{CONCLUS IONES}

Una buena planificación de la gestión de alcance permite determinar los requisitos, controlar $\mathrm{y}$ validar el alcance de un proyecto de implementación de sistemas MES. El modelo de planificación puede ser mejorado para dar respues ta a proyectos que busquen no solo mejorar los procesos productivos a través de la implementación de un sistema MES, sino que además tiendan a la eficiencia energética de los procesos productivos de una planta. El modelo de planificación presentó los siguientes inconvenientes una vez aplicado en la prueba piloto:

- El modelo tiene en cuenta los tiempos de entrega por parte del contratista, pero desconoce las fechas de aceptación o recepción del proyecto por parte de la empresa contratante

- El modelo no tiene en cuenta los procesos administrativos propios de una empresa de ingeniería de proyectos de automatización, en la cual se manejan varios proyectos a la vez.

\section{REFERENCIAS}

Project Management Institute. (2013). A guide to the project management body of knowledge. Pennsylvania PMI 1, 18-188.
Ailin Orjuela, L. A. (2011). Scope Planning in software projects. Revista Colombiana de Tecnologías de Avanzada, 2 (18), 62.

Banerjee, A. N. (2013). Design of Manufacturing Execution System for FMCG Industries. International Journal of Engineering and Technology (IJET), 5 (3), 2366-2367.

Brandenburg, P. (2013). Keys to integrating automation, MES, and business systems. In Tech Factory Automation, 2.

Chris Wubbolt, J. T. (2012). Considerations for Validation of Manufacturing Execution Systems. Journal of Validation Technology, 8084.

Christine Lesher, E. P. (2006). Applying a Phased Approach for a Successful Manufacturing Execution System (MES) Project. MESACitec.6.

Clemons, J. (7 de 12 de 2013). Maverick Technologies. Recuperado el 04 de 03 de 2014, de

http://www.mavtechglobal.com/ideas/2013/12/

$17 /$ some-pers pectives-on-mes-implementationspart-1/

D E Anisimov, I. S. (2011). Management Aspect in MES Implementeción Project. Automatión and Remote Control, 72 (6), 1319-1332.

Elliott, R. (2013). Manufacturing Execution System (MES) An Examination of Implementation Strategy. San Luis Obispo: California Polytechnic State University.26, 36.

Engelbrecht, D. (2008). Barriers to entry for MES. EngineerIT, 15-16.

Ferrazzi, A. (2012). Manufacturing execution system: a case study in the aerospace industry. Padua: University of Padua, 52-60.

Giunchetti, F. F. (2004). Coordenação de projetos para implementação de sistemas MES. Itajubá: Universidade Federal de Itajubá - unifei.51-62.

ISA. (2010). ANSI/ISA-95.00.01-2010 (IEC 62264-1 Mod). Enterprise-Control System Integration Part 1: Models and Terminology (Vols. ISBN: 978-1-936007-47-9). North Carolina: ISA.21-22.

Jason Knight, S. L. (10 de 01 de 2006). Medical Divece and Diagnostic Industry. Recuperado el 04 de 03 de 2014, de http://www.mddionline.com/article/s electingand-using-manufacturing-execution-system

Johnson., F. K. (2009). Building Your MES Team. Southwest Decision Sciences Institute, 5-8.

Kletti, J. (2007). Manufacturing execution System MES. Alemania: Springer, 81-117.

Kozletsov, A. (2012). MES - not only production planning production Automation. $\mathrm{R}$ a $\mathrm{t}$ i o $\mathrm{n}$ a 1 Enterpris e M a n a e m e n t, 6, 62,63. 
Larsen, K. M. (2009). What makes MES project a Sucess? MESA Europe Conference. Berlin.4451.

Lesher, C. P. (2009). Applying a Phased Approach for a Successful Russian Language Translation. En MESA White Paper \#19 (pág. 1). MESA ORG.3-10.

Project Management Institute. (2006). Practice Standard for Work Breakdown Structures. (Second, Ed.) Pennsylvania: Project Management Institute, 2-7.
Project manager Alliance. (16 de 10 de 2013). MES project management experience when requirements change. (Project manager Alliance) Recuperado el 04 de 03 de 2014, de http://www.mypm.net/articles/show_articles_co ntens .asp? articleID $=26351$

Shrivastava, A. (2013). Best Practices for Global MES Rollouts. New Jersey: Cognizant.4, 6.

The Institute of Electrical and Electronics Engineers. (2009). IEEE Recommended Practice forSoftware Requirements Specifications. IEEE Std 830 TM-1998 (R2009). New York: IEEE.1-2

Turley, F. (2009). El Modelo de Procesos PRINCE2. Una magnífica introducción a El Modelo de Procesos PRINCE2. 